\title{
Research on Morphology of Human Red Blood Cells (RBCs) in Different Gravity Fields
}

\author{
Lin LI, Shuang-Yin GAO* \\ Hospital of Wuhan University of science and technology, Wuhan Hubei Province, \\ China, 430081 \\ ${ }^{*}$ Corresponding author
}

Keywords: Human red blood cells (RBCs), Gravity fields, Morphology, Death rate.

\begin{abstract}
By testing fresh human red blood cells (RBCs), the morphology of human RBCs in different gravity fields was observed by SK2109P microscope. And the death rate of RBCs in different gravity fields were counted Results show that: RBCs can endure a certain gravity field. RBCs barely died under gravity fields less than $340 \mathrm{~g}$. The death rate increased from $40 \%$ to $70 \%$ while the gravity field increased from $1360 \mathrm{~g}$ to $1850 \mathrm{~g}$. When gravity field reached $3050 \mathrm{~g}$, the death rate became over $90 \%$, it was hard to find survival cells in viewing field.
\end{abstract}

\section{Introduction}

Supergravity can be obtained by centrifugal round and has been extensively used in cropper seed selected, microorganism variation [1-3], and it is also significant to medical research. Caused by launching and returning spacecrafts, the supergravity attracted interest of researchers after Apollo successfully landing on the moon. Natalia M. Arzeno [4] found cardiovascular deconditioning was still evident 6 days after returning from short-duration spaceflight, and parasympathetic modulation was diminished and sympathovagal balance was increased. Nadège Minois [5] reported the age shortened while prolonged exposed in the supergravity fields. Considering the effect on human body of supergravity fields, researchers have studied the countermeasure. Ana Diaz [6] designed a compact rotating centrifuge to research physiological deconditioning during space missions by making an artificial supergravity. The similar research worked by Yifan Yang [7] showed that artificial gravity is a potential countermeasure against the microgravity in space.

$\mathrm{RBCs}$ is the mian part of blood, their role was transport oxygens to support the life. Therefore, morphology of RBCs in supergravity fields has a direct influence on human supergravity training. This paper used human rad blood cells (RBCs) as an object, to research the effect of the supergravity on morphology of RBCs.

\section{Materials and Methods}

Choose fresh human RBCs as test materials and deal with rotating speed adjustable centrifuge (LD4-2A), which radius is $13.5 \mathrm{~cm}$ and the maximum rotational speed is 4500r/min. Observe morphology and count the death rate of RBCs dealt with and without spuergravity fields. The equation between rotational speed and the gravity is as shown in Eq 1.

$$
\mathrm{G}=\sqrt{\mathrm{g}^{2}+\mathrm{g}^{2}}=\sqrt{\mathrm{g}^{2}+\left((2 \pi \mathrm{n})^{2} \mathrm{r}\right)^{2}}
$$


$\mathrm{g}$ is the normal gravitational acceleration, $\mathrm{r}$ is the radius of the centrifuge, $\mathrm{n}$ is the rotational speed. $G$ is the super gravitational acceleration. The calculated result was showed in table 1 .

\section{Results and Discussion}

Fig.1 shows morphology of RBCs under different gravity fields. Fig.1(a) is the morphology of RBCs under the normal gravity. It shows that RBCs are smooth sphere with dark centre because the normal PBCs are concave in the centre which decreases the light reflection.

Under the $37 \mathrm{~g}$ supergravity field, morphology of partial BRCs changed, cell membrane shrank and resulted to a rugged cell surface. The proportion of deformed cells increased when the supergravity field increased to $150 \mathrm{~g}$. To $340 \mathrm{~g}$, the level of deformation increased but no dead cell was observed. Under 600g supergravity, RBCs died but healthy RBCs can be still observed, so death is not a common phenomenon.

The supergravity fields raised to $940 \mathrm{~g}, 1360 \mathrm{~g}, 1850 \mathrm{~g}$ and $2420 \mathrm{~g}$, the death rate of RBCs increased and large number of dead RBCs can be observed in viewing field. Meanwhile, survival RBCs deformed and healthy RBCs disappeared. When the supergravity reached $3050 \mathrm{~g}$, it was hard to find survival RBCs.

The death rates of RBCs in different gravity fields were count with 15-20 viewing fields, and the result was showed in Fig. 2. The death rate was $30 \%$ in $600 \mathrm{~g}$ supergravity field, and it increased slowly to $40 \%$ in $1360 \mathrm{~g}$ supergravity field with unapparent amplification. The death rate increased to $60 \%$ suddenly in $1850 \mathrm{~g}$ supergravity field, Then it increased rapidly with gavity increased. In $3050 \mathrm{~g}$ the death rate was $90 \%$, there were barely survival RBCs.

RBCs are consist of water and hemoglobin. Under supergravity, the molecular weight of $\mathrm{H}_{2} \mathrm{O}$ is less than the hemoglobin's according to Newton's laws .So the gravity dehydrates RBCs firstly which caused deformation and shrink. However, deformation will be recovered and RBCs survive. RBCs maybe die from hemoglobin dissolution(hemolytic phenomenon) because gravity slits cell membranes[9-10]. In this experiment, RBCs deformed but survived in the lower supergravity fields(less than $340 \mathrm{~g}$ ) because of dehydration, and cell membranes shrinks. As the supergravity field increased to a certain value $(600 \mathrm{~g}-1300 \mathrm{~g})$, cell membranes became slited and RBCs died from emoglobin dissolution. In this process, death rate raised slowly for part of cell membranes have not be slited. To $1850 \mathrm{~g}$, cell membranes were slited heavily, so the death rate increased rapidly. In the end, all of RBCs died.

\section{Conclusion}

RBCs can endure a certain level of supergravity. They can survived in the supergravity field less than $340 \mathrm{~g}$, and dead cells were observed from $600 \mathrm{~g}$. The death rate increased slowly untill $1360 \mathrm{~g}$, and increased rapidly in $1850 \mathrm{~g}$. Since then, with the increase of supergravity field, the death rate increased continuously. 



Fig.1 Mophology of RBCs in different supergravity fields

(a) $0 \mathrm{~g}$ (b) $37 \mathrm{~g}$ (c) $150 \mathrm{~g}$ (d) $340 \mathrm{~g}$ (e)600g (f) $940 \mathrm{~g}$ (g)1360g (h) $1850 \mathrm{~g}$ (i) $2420 \mathrm{~g}$ (j) $3050 \mathrm{~g}$ 


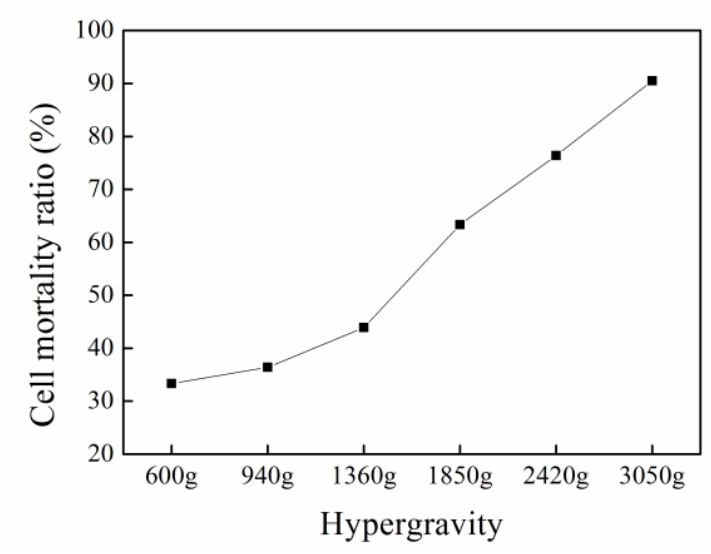

Fig.2 Death rate of different supergravity fields

\section{References}

[1] Hodick D, Sievers A. Hypergravity can reduce but not enhance the gravitropic response of Chara globularis protonemata [J]. Protoplasma, 1998, 204: 145-154.

Reference to a book:

[2] Fitzelle, K.J, Kiss, J.Z. Restoration of gravitropic sensitivity in starch-deficient mutants of Arabidopsis by hypergravity [J]. Journal of experimental botany, 2001, 355(52): 265-275.

[3] Takayuki Hoson, Kazuyuki Wakabayashi. Role of the plant cell wall in gravity resistance. Phytochemistry 2015, 112: 84-90.

[4] G. Kim Prisk, Rui Carlos Sá, Chantal Darquenne, Cardiogenic mixing increases aerosol deposition in the human lung in the absence of gravity $[\mathrm{J}]$. Acta Astronautica, 2013, 92(1): 15-20.

[5] Nadège Minois The hormetic Effects of Hypergravity on Longevity and aging [J]. Dose Response, 2006, 4(2): 145-154.

[6] Ana Diaz, Chris Trigg, Laurence R. Young, Combining ergometer exercise and artificial gravity in a compact-radius centrifuge [J]. Acta Astronautica, 2015, 113: 80-88.

[7] Yifan Yang, Michael Baker, Scott Graf etc. Hypergravity resistance exercise: the use of artificial gravity as potential countermeasure to microgravity [J]. Journal of Applied Physiology, 2007, 103(5).

[8] Caiozzo VJ, Rose-Gottron C, Baldwin KM, etc. Hemodynamic and metabolic responses to hypergravity on a human-powered centrifuge [J]. Aviat Space Environ Med, 2004, 75(2):101-108.

[9] A.S. Ademiloye, L.W. Zhang, K.M. Liew. Numerical computation of the elastic and mechanical properties of red blood cell membrane using the higher-order Cauchy-Born rule, Applied Mathematics and Computation, 2015, 268(1): 334-353.

[10] Prabhakar Deonikar, Mahendra Kavdia. Contribution of membrane permeability and unstirred layer diffusion to nitric oxide-red blood cell interaction [J], Journal of Theoretical Biology, 2013, 317:321-330. 